\title{
Perfil de la consulta de urgencia infantil en el Hospital Regional Rancagua 2002-2003
}

\author{
HEIDY LEIVA(1), MARCELA ORTEGA(2), CAROLINA MARTINOVIC(2)
}

\section{RESUMEN}

El propósito del estudio es contribuir a la mejor gestión de la red asistencial del Servicio de Salud de la Sexta Región, mediante el conocimiento de las caracteristicas y pertinencia de la atención de urgencia infantil en el Hospital Regional de Rancagua, a través de los siguientes objetivos: a) Determinar el volumen de consultas al Servicio de Urgencia de menores de 15 años; b) Conocer las características de la población consultante en las variables edad, sexo, comuna de residencia; c) Medir la distribución mensual de las consultas y determinar si existe estacionalidad; d) Conocer los motivos de consulta declarados por los padres y relacionarlos con la pertinencia, usando como una aproximación a la pertinencia o gravedad, la decisión de hospitalización; e) Conocer el destino de los pacientes derivados desde otros establecimientos de la red asistencial de la región al hospital base, mediante una interconsulta. Se trata de un estudio descriptivo de las consultas de urgencia de menores de 15 años durante los años 2002 y 2003 en el Servicio de Urgencia del Hospital Regional Rancagua. El motivo de consulta principal es la fiebre en el 29,72\% de la consulta pediátrica y en la consulta quirúrgica infantil el motivo de consulta más frecuente es contusión de extremidad superior (21,6\%). Se hospitaliza el 9,9\% del total de consultas pediátricas y el $6,1 \%$ de los pacientes que consultan por causas quirúrgicas. Existe un gran numero de consultas derivadas desde la atención primaria y que no fueron causa de hospitalización por lo que se estima que en su mayoría debieron ser resueltas en ese nivel de atención, ya que no podemos desconocer las importancia que tienen ciertas consultas que siendo urgentes no son graves, como la epistaxis y que en este estudio no se han desglosado.

Palabras clave: urgencia infantil, patología pediátrica.

\section{ABSTRACT \\ PROFILE OF THE CHILDREN'S EMERGENCY SERVICE IN THE RANCAGUA REGIONAL HOSPITAL, 2002-2003}

This study serves the purpose of contributing to the management of the medical network of the Sixth Region Health Service through acquaintance with the characteristics and relevance of the Children's Emergency Service health care in the Rancagua Regional Hospital, by means of the following objctives: a) Ascertaining the volume of Emergency Service visits of children under 15 ; b) Identifying the characteristics of the consulting population in the variables of age, sex, commune of residence; c) Assessing the monthly distribution of visits and determining if there is

\footnotetext{
(1) Hospital Regional Rancagua. Servicio de Pediatría. draheidy@terra.el

(2) Intenas. Faculad de Medicina. Universidad de Santiago de Chile.
} 
seasonality; d) Identifying the cause of the visit declared by the parents and relating it to pertinence, using as an approximation to pertinence or seriousness of the condition, the decision of hospitalization; e) Being informed of the destination of patients that are being derived from other establishments of the regional medical network to the base hospital, by means of intrahospital consultations. This is a descriptive study of the emergency visits of minors under 15 years of age during the years 2002 and 2003 to the Emergency Service of the Rancagua Regional Hospital. The main cause for visits is fever in $29.72 \%$ of pediatric visits and in children's surgical visits the most frequent reason for consulting is contusion of upper extremity (21.6\%). There exists a great number of visits derived from primary attention and which required no hospitalization; it is therefore deemed that in the majority of cases these must have been resolved at that level of attention, since we cannot disregard the importance of certain consultations which, although urgent, are not serious, such as epistaxis, which has not been considered.

Key words: Child emergencies, Pediatric pathologies.

\section{INTRODUCCIÓN}

Una urgencia médica es toda situación que requiera una acción médica inmediata. En Chile, casi la mitad de todas las atenciones de salud que se brindan en un año, sumadas las del sector público y las del sector privado corresponden a las otorgadas en los servicios de urgencia del país, según estadísticas del Instituto Nacional de Estadísticas. Suman 13 millones de atenciones anuales.

Es importante citar a la vez, que el Servicio de Urgencia Pediátrica del Hospital Regional Rancagua constituye el principal centro de derivación de la Sexta Región, abarcando una población infantil de 209.290 habitantes menores de 15 años ${ }^{1}$.

La situación de la atención de los Servicios de Urgencias del Sistema de Salud Público, es objeto de creciente preocupación de la comunidad sanitaria y de la población en general, debido al gran número de consultas, a la amplia gama de patologías consultantes, desde las más simples, hasta aquéllas que podrian comprometer la vida del paciente, junto con citar que constituye la principal vía de ingreso a los Servicios Hospitalarios. Es por esto, que consideramos de vital importancia realizar un análisis que nos revele la situación actual de nuestro servicio de urgencia.

Debemos considerar que la atención de Urgencia está dada en un contexto en el cual el contacto médico-paciente, se desarrolla bajo circunstancias imprevistas y a veces apremiantes, generando así, una situación que compromete en forma significativa la afectividad tanto del niño como de su núcleo familiar.

Para asegurar una atención eficiente y resolutiva a la población que consulta a nuestro servicio de urgencia, debemos estar al tanto de cuáles son las patologías que afectan a nuestra comunidad y que llevan a consultar a los pacientes, en espera de una adecuada atención. Para conocer el perfil de morbilidad de los niños en la Sexta Región, se ha analizado previamente la atención cerrada en las camas pediátricas públicas y privadas de la región ${ }^{2}$ y las consultas de especialidad del Hospital Regional Rancagua ${ }^{3}$.

Un segundo problema a considerar es el uso inadecuado de los servicios de urgencia que se ha acentuado en forma creciente en el último tiempo, sobrecargando al personal asistencial con patologías que no requieren atención inmediata o bien corresponden a afecciones que deberían resolverse en la atención primaria, lo que conlleva a un gasto innecesario y no presupuestado de los recursos disponibles ${ }^{4,5}$.

\section{MATERIAL Y MÉTODO}

El estudio es de tipo descriptivo. El universo en estudio es el total de consultas de urgencia de menores de 15 años en los años 2002 y 2003 
del Hospital Regional Rancagua que alcanzan a 117.806. La fuente de información son los Datos de Atención de Urgencia (DAU) que son ingresados en el sistema IBM, extrayendo los datos a un archivo plano y convertido posteriormente a una base de datos Access. El DAU no tiene digitada la hipótesis diagnóstica del médico de urgencia en el archivo IBM, lo que constituye una limitación de este estudio.

Esta base se analiza mediante Epilnfo 2001, $\checkmark$ 3.2.2. Las variables consideradas fueron: edad, sexo, comuna de residencia, sección de urgencia de atención (Adulto-Cirugía Infantil o Pediátrica-Dental), mes de atención, motivo de consulta y destino.

Definiciones operacionales: a) Consulta de Urgencia Pediátrica: atención de urgencia a niños cuyos padres no declaran motivos de consulta susceptibles de eventual procedimiento quirúrgico; b) Consulta de Urgencia Quirúrgica: atención de urgencia infantil por motivos de consulta que llevan a eventual procedimiento quirúrgico. Se realiza en el Servicio de Urgencia de Adultos del Hospital Regional Rancagua; c) Interconsulta: derivación desde otro establecimiento de salud, de la atención primaria municipal, de otro hospital del Servicio de Salud o desde una consulta médica privada; d) Debido a que no existe registro de la hipótesis diagnóstica que hace el médico de urgencia, se usa como un indicador de gravedad o pertinencia, la hospitalización del paciente. Al respecto, estamos conscientes de que existen consultas en el servicio que siendo urgentes no son graves, como una epistaxis, o una herida contusa que necesite sutura. En ambas se justifica la consulta al servicio de urgencia. En este estudio no se separaron estos casos.

Tabla 1

\begin{tabular}{lrr}
\hline Grupo etáreo & \multicolumn{1}{c}{$\mathbf{n}$} & \multicolumn{1}{c}{$\%$} \\
\hline Menor o igual a 1 año & 7.795 & 8,4 \\
Mayor de 1 año y & 33.958 & 35,5 \\
menor de 6 años & & \\
6 y más años & 32.362 & 33,9 \\
Total & 95.576 & 100 \\
\hline
\end{tabular}

\section{RESULTADOS}

Se presentan separadamente las atenciones de Urgencia Adulto-Cirugía Infantil y las atenciones de la Urgencia Pediátrica. Estas últimas, incluyen la atención del odontólogo a menores de 15 años, que es de baja frecuencia, menos del $4 \%$.

Urgencia pediátrica: El universo es de 95.576 consultas.

1) Distribución por sexo y edad: $53,5 \%$ son varones (49.528 consultas) y $43.074(46,5 \%)$ consultas de niñas. La diferencia entre ambos sexos es estadísticamente significativa, $\mathrm{p}=$ 0,0000 (Tabla 1).

El $8,4 \%$ de las consultas ( 7.795 atenciones) corresponde al grupo de menores de 1 año.

2) Distribución por mes de consulta: La frecuencia mayor de consultas se observa en el mes de julio con 9.101 consultas $(9,5 \%)$, coincidente con el aumento de las enfermedades respiratorias en la infancia en los meses de invierno. La menor frecuencia se observa en febrero que tiene 6.250 consultas $(6,5 \%)$. El promedio diario de atenciones es de 261,8 .

3) Distribución por comuna de residencia: El 99,1\% (94.710 consultas) de los pacientes vive en las comunas de la Provincia de Cachapoal, correspondiendo el 72,2\%(68.815 consultas) a niños de la comuna de Rancagua, y en segunda frecuencia consultan pacientes provenientes de la comuna de Machalí.

Tabla 2

\begin{tabular}{lrr}
\hline Motivo & n consultas & \multicolumn{1}{c}{$\%$} \\
\hline Fiebre & 27.449 & 29,72 \\
Vónitos & 10.267 & 10,74 \\
Tos & 5.850 & 6.12 \\
Interconsulta & 5.583 & 5,78 \\
Dificulad para respirar & 4.980 & 5,21 \\
Resfrio & 3.309 & 3,46 \\
Dolor abdominal & 4.647 & 4,86 \\
Dolor no especificado & 4.017 & 4,2 \\
Alergia & 2.910 & 3,04 \\
Diarrea & 2.709 & 2,93 \\
Erupción cutánea & 1.164 & 1,22 \\
Convulsiones & 355 & 0,37 \\
Resto & 23.023 & 24,1 \\
Total & 95.576 & 100 \\
\hline
\end{tabular}


Trescientos cincuenta y dos niños vienen desde comunas de Colchagua y 58 lo hacen desde la provincia de Cardenal Caro.

4) Motivo de consulta: La fiebre tiene la primera frecuencia, $29,72 \% \quad(27.449$ consultas), seguido de los vómitos con $10,74 \%$ (10.267 atenciones). A continuación se observan la tos, $6,12 \%(5.850)$ y las interconsultas $5,21 \% \quad(5.583$ consultas $)$ (Tabla 2).

5) Distribución de las interconsultas por comuna de residencia: El total de las atenciones que tienen una interconsulta como motivo de consulta es de $5.583(5,21 \%)$ y de éstas el 95,54\% (5.334 consultas) proviene de la provincia de Cachapoal. En el caso de las provincias de Colchagua y Cardenal Caro, también, se produce la derivación directa desde comunas como Chimbarongo o Pichilemu.

6) Hospitalización: Se analiza la población de niños hospitalizados luego de la consulta de urgencia pediátrica que es de 9.457, $(9,95 \%)$

Tabla 3

\begin{tabular}{lcc}
\hline Grupo etáreo & $\mathbf{n}$ & $\%$ \\
\hline Menor o igual a 1 año & 460 & 4,9 \\
Mayor de 1 año y & & \\
menor de 6 años & 4.967 & 52,3 \\
6 y más años & 4.050 & 42,8 \\
Total & 9.457 & 100 \\
\hline
\end{tabular}

Tabla 4

\begin{tabular}{lrr}
\hline Motivo & n consultas & \% \\
\hline Fiebre & 2.697 & 28,5 \\
Vómitos & 976 & 10,3 \\
Tos & 600 & 6,3 \\
Interconsulta & 577 & 6,1 \\
Dificultad para respirar & 486 & 5,1 \\
Resfrio & 338 & 3,6 \\
Dolor abdominal & 437 & 4,6 \\
Dolor no especificado & 398 & 4,2 \\
Alergia & 326 & 3,4 \\
Dolor garganta & 265 & 2,8 \\
Dolor ó́dos & 231 & 2,4 \\
Convulsiones & 37 & 0,4 \\
Campana IRA & 124 & 1,3 \\
Resto & 1.965 & 20,8 \\
Total & 9.457 & 100 \\
\hline
\end{tabular}

del total de consultas en el mismo período.

7) Distribución de hospitalizados por grupo etáreo (Tabla 3).

Se aprecia mayor hospitalización en preescolares y escolares. El promedio de edad de los niños hospitalizados luego de una consulta de urgencia es de 5,7 años con una ds de 3,58 , p $25=3,0$ y p 75 es de 8,0 años.

\section{8) Distribución de hospitalizados por motivo} de consulta (Tabla 4).

La distribución del motivo de consulta de los pacientes hospitalizados es similar a la encontrada en el total de los consultantes, aunque se aprecia un menor grado de dispersión de motivos de consulta. La fiebre es, en los 3 grupos etáreos, la mayor causa de consulta. Representa el 17\% (78 niños) de las causas de hospitalización en los menores de 1 año; el $31,7 \%$ (1.575) de los ingresos de 1 a 6 años y el 26\% (1.053) en los escolares internados. La interconsulta aparece en el 14,8\% (68 pacientes) del grupo de menores de 1 año, y los vómitos en el $8 \%$ ( 37 niños). La tos es causa de consulta en el 8\% (37) de los menores de 1 año; $7,5 \%$ (373 atenciones) de los mayores de 1 año y menores de 6 años y en el 4,8\% (194 niños) de los escolares. El análisis de Chi cuadrado demuestra una $\mathrm{p}=0,0000$, es decir, los niños hospitalizados son diferentes en el motivo de consulta según el grupo etario.

Urgencia quirúrgica infantil: El universo es de 22.230 atenciones.

1) Distribución por sexo: En 21.885 se registrar la información del sexo, constatándose 13.257 consultas de varones $(60,6 \%)$ y 8.628 niñas $(39,4 \%)$. En la consulta de urgencia quirúrgica hay mayor frecuencia de consultantes varones, diferencia que es estadísticamente significativa $\mathrm{p}=0,0000$.

Tabla 5

\begin{tabular}{lrc}
\hline Grupo etáreo & $\mathbf{n}$ & $\%$ \\
\hline Menor o igual a 1 año & 1.249 & 5,6 \\
Mayor de 1 año y & & \\
menor de 6 años & 5.433 & 24,4 \\
6y más años & 15.548 & 69,9 \\
Total & 22.230 & 100 \\
\hline
\end{tabular}


2) Distribución por edad: El grupo escolar presenta un predominio de las consultas de urgencia quirúrgica (Tabla 5).

3) Distribución por mes de consulta: El promedio de consultas diario es de 61 . El mes de más consultas es octubre con 2.266 atenciones ( $10,2 \%$ del total), seguido de noviembre con $2.238(10,1 \%)$. Los meses con menos atenciones corresponden a las vacaciones, enero y febrero $(1.610$ y 1.469 respectivamente).

4) Distribución por comuna de residencia:

21.808 consultas, $98,1 \%$, corresponden a niños que viven en comunas de Cachapoal. El 66,2\% de los niños provienen de la comuna de Rancagua. Se registran $242(1,1 \%)$ atenciones a pacientes de las provincias de Colchagua y Cardenal Caro. Es preciso señalar que en el Hospital de San Fernando existe la subespecialidad Cirugía Infantil que puede recibir la derivación de estas provincias.

5) Motivo de consulta: Los motivos de consulta están directamente asociados a traumatismos y accidentes escolares. La interconsulta es también un motivo frecuente (Tabla 6).

6) Hospitalización: Se analiza la población de niños hospitalizados luego de la consulta en urgencia de Cirugía Infantil que es de 1.362, lo que representa el $6,1 \%$ del total de consultas en el mismo período.

7) Distribución de hospitalizados por grupo

Tabla 6

\begin{tabular}{lrr}
\hline Motivo & n consultas & $\%$ \\
\hline Accidente escolar & 2.527 & 11,4 \\
Interconsulta & 3.689 & 16,6 \\
Control paciente hospitalizado & 1.112 & 5,0 \\
Contusión de cabeza & 1.508 & 6,8 \\
Contusión extremidad inferior & 2.361 & 10,6 \\
Contusión extremidad superior & 4.812 & 21,6 \\
Dolor abdominal & 612 & 2,8 \\
Mordedura de perro & 533 & 2,4 \\
Quemaduras & 430 & 1,9 \\
Fractura extremidad superior & 55 & 0,2 \\
Fractura extremidad inferior & 45 & 0,2 \\
Constatación lesiones & 525 & 2,4 \\
Accidente trânsito & 273 & 1,2 \\
Resto & 3.748 & 16,9 \\
Total & 22.230 & 100 \\
\hline
\end{tabular}

etáreo: Existe un predominio de la hospitalización de escolares, similar al encontrado en la distribución de las consultas, destacando el escaso número de lactantes menores de 1 año que consultan por patologías quirúrgicas (Tabla 7).

8) Distribución de hospitalizados por motivo consulta: La distribución del motivo de consulta de los pacientes hospitalizados sigue la misma distribución de las consultas (Tabla 8).

Al comparar los preescolares y escolares que son hospitalizados, en los motivos de consulta más frecuentes, no se aprecian diferencias significativas.

Por lo tanto:

1) La consulta de urgencia pediátrica es de alta frecuencia, con un promedio de 261 consultas diarias en el año, con evidente estacionalidad en que las enfermedades respiratorias hacen aumentar en forma estadísticamente significativa las consultas en 3 meses, en especial en el mes de julio.

2) Las características de los pacientes consultantes por motivos pediátricos y

Tabla 7

\begin{tabular}{lrc}
\hline Grupo etáreo & n & \% \\
\hline Menor o igual a 1 año & 9 & 0,7 \\
Mayor de 1 año y & & \\
Menor de 6 años & 312 & 22,9 \\
6 y más años & 1.041 & 76,4 \\
Total & 1.362 & 100 \\
\hline
\end{tabular}

Tabla 8

\begin{tabular}{lrr}
\hline Motivo & n consultas & \% \\
\hline Accidente escolar & 133 & 9,8 \\
Interconsulta & 264 & 19,4 \\
Contusión de cabeza & 103 & 7,6 \\
Contusión extremidad inferior & 140 & 10,3 \\
Conusión extremidad superior & 242 & 17,8 \\
Dolor abdominal & 42 & 3,1 \\
Mordedura de perro & 41 & 3,0 \\
Quemaduras & 10 & 0,8 \\
Fractura extremidad superior & 2 & 0,1 \\
Fractura extremidad inferior & 1 & 0.1 \\
Accidente tránsito & 10 & 0,7 \\
Resto & 374 & 27,4 \\
Total & 1.362 & 100 \\
\hline
\end{tabular}


quirúrgicos son diferentes.

3) Los pacientes atendidos en Urgencia Pediátrica son mayoritariamente niños, con un $30 \%$ de lactantes y provienen en $95,5 \%$ de las comunas de Cachapoal.

4) La gravedad y, por ende, la pertinencia de las consultas, por tratarse de atención de urgencia, es discutible, lo que queda demostrado con la baja tasa de hospitalización que alcanza al $9,9 \%$ de la consulta pediatra y el $6,1 \%$ de las consultas quirúrgicas infantiles.

5) Aparece nítidamente que los consultantes son, en la gran mayoría, de la provincia de Cachapoal y se puede deducir que el Hospital Rancagua se comporta, en la urgencia de los niños, como un hospital secundario de derivación de esta provincia.

6) La interconsulta concluye con la hospitalización del paciente derivado en baja proporción, lo que permite concluir que el contacto previo con un profesional de la salud en otro establecimiento no mejora la pertinencia de la consulta.

\section{DISCUSIÓN}

1) El funcionamiento de la red de atención del servicio de salud debe ser analizado acuciosamente, considerando que existen derivaciones desde hospitales que deberían consultar en otros establecimientos antes del hospital regional.

2) El Servicio de Urgencia del Hospital Regional funciona como un establecimiento secundario de la provincia de Cachapoal, según muestra la distribución por comuna de residencia de los consultantes y por la baja tasa de hospitalización. Sin embargo, al interior de esta provincia y en la micro área de Rengo no debería existir derivación directa desde comunas como Las Cabras o Malloa, que tienen como lugar de derivación más cercano a los hospitales de San Vicente y Rengo.

3) La información analizada permite sugerir que un gran número de consultas debería haber sido resuelta en el nivel primario.
Seria interesante determinar si la decisión de las personas al traer a sus hijos al Hospital Regional, de urgencia, revela insuficiencia de los consultorios de atención primaria por falta de resolutividad o por déficit de oferta de horas médicas. Como sólo el $5,78 \%$ de las consultas tiene como motivo una interconsulta, es decir, existió un contacto con algún profesional de la atención primaria previo, la consulta surge de la búsqueda de una alternativa distinta de la APS.

4) La implementación de 2 SAPU en la comuna de Rancagua parece ser una alternativa adecuada para disminuir la consulta no justificada, pero debe evaluarse permanentemente en forma coordinada con el Hospital Regional para optimizar su funcionamiento y evitar la duplicidad de consultas.

5) La extensión horaria de la APS debe funcionar con todos los recursos diagnósticos y terapéuticos de forma de ser capaz de resolver integralmente las patologías a ese nivel.

6) La comunidad debe recibir educación permanentemente acerca del uso correcto del sistema de salud y, especialmente, los dirigentes vecinales pueden transformarse en aliados poderosos en la información a Ios vecinos.

\section{REFERENCIAS}

1.- INSTITUTO NACIONAL DE ESTADÍSTICAS. Censo 2002. Chile.

2.- HOSPITAL REGIONAL RANCAGUA. Base datos Consulta del Consultorio Adosado de Especialidades $2002-2003$.

3.- DEPARTAMENTO ESTADÍSTICAS SERVICIO SALUD O'HIGGINS. Base datos egresos hospitalarios Sexta Región $2001-2002$.

4.- PEIRÓ S, SEMPERE T, OTERINO D. Efectividad de las Intervenciones para Reducir la Utilización Inapropiada de los Servicios Hospitalarios de Urgencia, Revisando la Literatura 10 años después del Informe del Defensor del Pueblo. Informe de la Asociación de Economía de la Salud. AES. 1999.

5.- SOLIS F, MEDINA E. Realidad de la consulta médica de urgencia. Rev Méd Chile 1987; 115: 1210-7.

Usted puede comentar éste y otros artículos publicados en la Revista Chilena de Salud Pública, enviando un correo electrónico a revistasp@ med.uchile.cl 